\title{
Responsabilité et mesure, un rôle essentiel dans la prescription : le millepertuis en exemple
}

\author{
P. Goetz \\ (C) Lavoisier SAS 2015
}

La difficulté de proposer un traitement vient souvent du fait que le thérapeute n'agit pas en terrain vierge. Le (ou la) patient(e) a souvent déjà un traitement allopathique en place dont il faut tenir compte. Souvent il faut installer un traitement par phyto-aromathérapie en plus du traitement précédent et ne pas remplacer de but en blanc celui-ci. C'est surtout souvent le cas avec un antidépresseur : ne pas créer de syndrome de sevrage en supprimant l'antidépresseur. Celuici est néfaste au patient et de plus il est impossible de juger de l'efficacité du nouveau traitement.

Ceci est le cas bien particulier de l'utilisation du millepertuis dans un traitement d'une dépression légère ou moyenne ${ }^{1}$....

Ne jouons pas à l'apprenti sorcier pour autant. Dans certains cas comme le traitement du SIDA, un traitement concomitant de millepertuis n'est pas d'une grande utilité. Quand un sujet est sous anticoagulant comme la fluindione (Préviscan $^{(\mathbb{R}}$ ) ou la warfarine, le médecin est apte, grâce à des contrôles de l'INR, de doser l'anticoagulant en fonction des effets du millepertuis.

Pour les contraceptifs oraux, l'intolérance est surtout établie pour l'éthyniloestrdiol et le désogestrel, et il faudra impérativement tenir compte de ces médications lors de la prescription du millepertuis. Nous nous rappellerons que l'effet des contraceptifs peut être inhibé par d'autres médicaments.

In vivo, chez des patients, nous connaissons le fait que rajouter un antidépresseur à un premier souvent en augmente l'effet. Par expérience nous avons noté qu'une petite dose de millepertuis certifié standardisé en hyperforine, peut augmenter une amitryptiline ou un IRS, sans qu'il y ait de syndrome sérotoninergique, mais une simple augmentation des effets.

P. Goetz $(\bowtie)$

Dumenat de phytothérapie, Paris-XIII,

F-93017 Bobigny cedex, France

e-mail : paul.goetz@wanadoo.fr

\footnotetext{
${ }^{1}$ Lire en pages intérieures la mise au point sur les interactions
} médicamenteuses bénéfiques et dérangeantes du millepertuis
De ce fait il est important que le maniement du millepertuis soit réalisé par un professionnel en fonction des circonstances. Il est tout aussi primordial que le pharmacien qui conseille ou déconseille ce type de produit soit bien informé et que, dans certains cas, il discute d'une coprescription de son intérêt avec le prescripteur.

Souvent les auteurs terminent une monographie par « plantes à associer », et les différentes instances (WHO, Escop, Commission E, EMA) précisent pour chaque monographie « si rien n'est prescrit par ailleurs ». Faire de la monothérapie (c'est-à-dire avec une seule drogue), relève souvent de l'utopie quoiqu'il existe bien des cas où cela peut être suffisant.

Les associations doivent apporter des plantes agissant dans le même sens mais avec des modalités différentes. Inutile d'ajouter de l'éleuthérocoque à du ginseng, ou de l'aubépine à de la digitale, etc. Par contre associer Echinacea à Eupatorium perfoliatum et du Baptisia tinctoria est judicieux puisque le but est de stimuler tous les éléments immunocompétents, ou presque, la défense de l'organisme anti-infectieuse par la voie immunologique.

Le danger d'association de phytomédicament à une thérapie conventionnelle réside surtout dans les cas où on rajoute un antidépresseur efficace comme le millepertuis à un antidépresseur classique, ou une drogue ayant un pouvoir fluidifiant (même si elle n'est pas prescrite pour cela : comme la reine des prés contre la douleur) à un fluidifiant sans tenir compte du taux d'INR. Beaucoup de thérapeutes n'ont pas conscience que la giflofle (Syzygium aromaticum) a un effet fluidifiant, donc prudence !

Notre revue se veut avant tout professionnelle et nous essayons, associés aux auteurs d'articles, de vous apporter cette qualité qu'il faut pour faire de la «médecine ». 